\title{
The Probabilistic Nature of Synaptic Transmission at a Mammalian Excitatory Central Synapse
}

\author{
B. Walmsley, ${ }^{1}$ F. R. Edwards, ${ }^{2}$ and D. J. Tracey ${ }^{1}$ \\ 'Neural Research Laboratory, School of Anatomy, University of New South Wales, Kensington, N.S.W., Australia, and \\ "Experimental Neurology Unit, John Curtin School of Medical Research, Australian National University, Canberra, A.C.T., \\ Australia
}

The synaptic connection between single group I afferents and dorsal spinocerebellar tract (DSCT) neurons in the cat spinal cord has been studied in an attempt to gain insight into the mechanisms of excitatory synaptic transmission in the mammalian CNS. Fluctuations in the amplitude of single group I fiber EPSPs in DSCT neurons were examined using a numerical deconvolution procedure to reduce the effects of contaminating noise. In general, it was found that single fiber EPSPs fluctuate in peak amplitude between discrete levels separated by equal or quantal increments.

Many previous studies have proposed simple binomial statistics as a general model of quantal synaptic transmission. In the present study we show that simple binomial statistics do not describe the fluctuations in amplitude of single group I fiber EPSPs in DSCT neurons. It is suggested that nonuniformities in the probability of transmitter release from release site to release site explain the failure of the binomial model to describe the EPSP fluctuation pattern at this synapse. Nonuniform quantal transmission is proposed as a more adequate description of excitatory synaptic transmission in the mammalian CNS.

The present study is aimed at understanding the probabilistic nature of synaptic transmission in the mammalian CNS. The use of the binomial model as a description of quantal synaptic transmission was first proposed by del Castillo and Katz (1954) for the neuromuscular junction. This model is appealing because of its inherent simplicity and because the binomial parameters $N$ and $P$ may have physical correlates. It is generally accepted that, at the neuromuscular junction, the quantal event or the quantal increment of the endplate potential is the result of release of transmitter from a single synaptic vesicle. The binomial parameter $N$ has been attributed to the number of quanta available for release or, alternatively, to the number of transmitter release sites. McLachlan (1978) postulated that " $N$ is the number of synaptic vesicles filled with transmitter located at release

Received Apr. 25, 1986; revised Sept. 22, 1986; accepted Sept. 25, 1986.

We are grateful to Drs. A. R. Martin, E. M. McLachlan, S. J. Redman, and R. J. Sayer for their comments and suggestions on this paper, and to Dr. J. Eccleston in the Mathematics Department, University of New South Wales, for useful discussions on statistical matters. We are also grateful to Dr. J. D. Clements for assistance with computer programs, and we would like to thank Ms. Madeleine

J. Nicol for typing the manuscript.

Correspondence should be addressed to Dr. B. Walmsley, Neural Research Laboratory, School of Anatomy, University of N.S.W., P.O. Box 1, Kensington, N.S.W., Australia.

Copyright (C) 1987 Society for Neuroscience $0270-6474 / 87 / 041037-10 \$ 02.00 / 0$ sites and activated by simultaneous presence of $3 \mathrm{Ca}^{2+}$ ions at some receptor." For the simple binomial model to apply, the quantal release probability, $P$, must be identical at all release sites.

The binomial model is useful not because of mathematical convenience, but because it enables the experimenter to distinguish between changes in the quantal content, the quantal size, and release probability following alterations in synaptic transmission (either naturally occurring or experimentally induced). For this reason many studies of synaptic transmission in the CNS have attempted to describe fluctuations in synaptic potentials using a simple binomial model (for reviews, see Martin, 1977; McLachlan, 1978). One of the major difficulties in these studies has been the presence of background noise obscuring the synaptic potential. A deconvolution procedure developed by Edwards et al. (1976) enabled the effects of such noise to be reduced and gave greater resolution to the amplitude fluctuation patterns of the synaptic potentials. This procedure, which has since undergone considerable refinement (Wong and Redman, 1980; Jack et al., 1981; Redman and Walmsley, 1983; Clements et al., 1986), has been used in the present study to examine the fluctuations in the amplitude of single group I fiber EPSPs evoked in dorsal spinocerebellar tract (DSCT) neurons.

In agreement with a previous study (Tracey and Walmsley, 1984), single fiber EPSPs were found to fluctuate in amplitude between discrete levels separated by equal, or quantal, increments. Anatomical studies (Tracey and Walmsley, 1984; Walmsley et al., 1985, 1987) have demonstrated that a single group I muscle afferent gives rise to a variable number of synaptic boutons contacting a DSCT neuron and that each bouton may contain a variable number of release sites. It was suggested that transmission between single group I muscle afferents and DSCT neurons occurs with quantal events corresponding to allor-nothing transmission at these synaptic release sites (Tracey and Walmsley, 1984). The present study demonstrates that fluctuations in the amplitude of single fiber EPSPs in DSCT neurons cannot be adequately described by simple binomial statistics. A likely explanation for the failure of the binomial model to describe the EPSP fluctuation pattern at this connection is that the probability of transmitter release differs from release site to release site. Such variation may occur from bouton to bouton or from release site to release site within the same bouton.

\section{Materials and Methods}

Experiments were performed on adult cats, induced and maintained on sodium pentobarbital anesthesia. Mean arterial pressure, expired $\mathrm{CO}_{2}$, and respiration rate were continually monitored. 


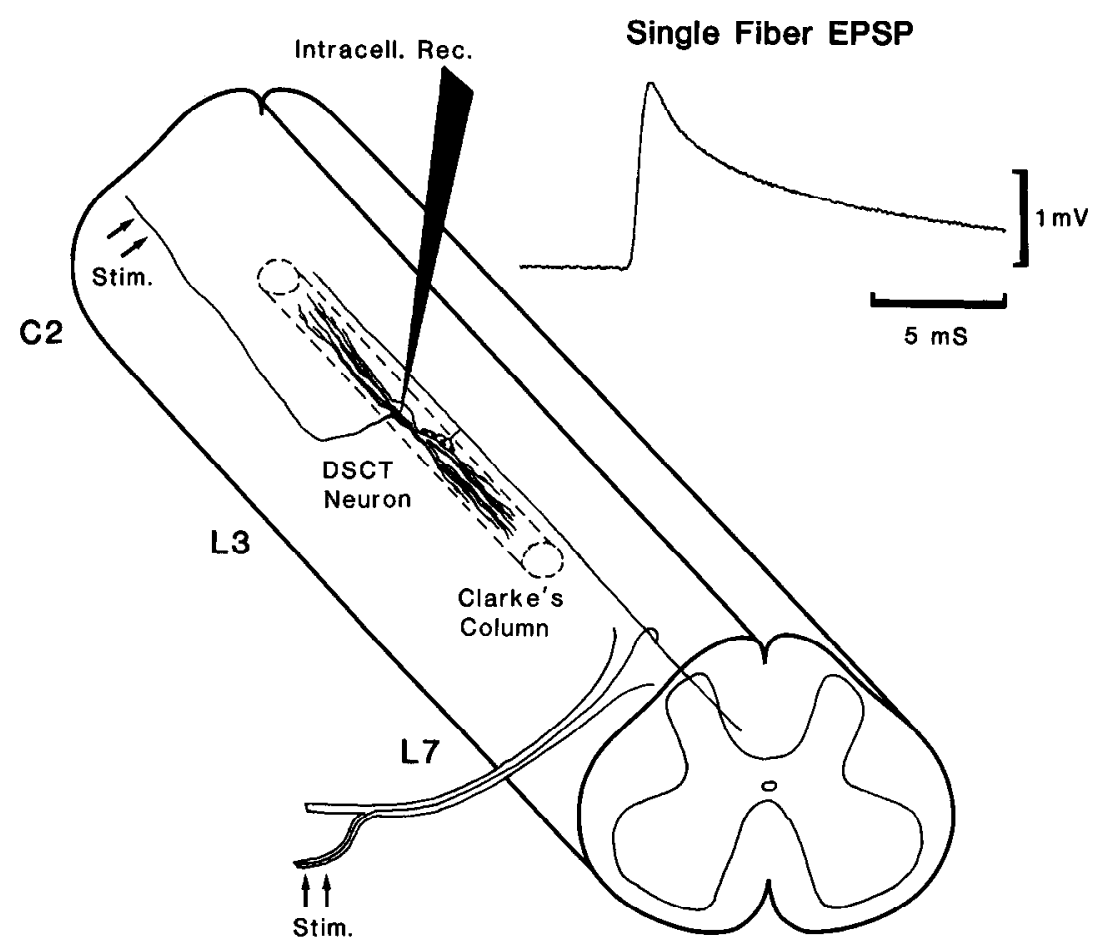

Figure 1. Experimental arrangement for intracellular recording of single group I fiber EPSPs in DSCT neurons in the upper lumbar region of the cat spinal cord. The average time course of one such EPSP is shown (upper right). Probability density distributions for the noise and the evoked EPSP (EPSP + noise) amplitudes were constructed from measurements in 4000 trials (lower plots).

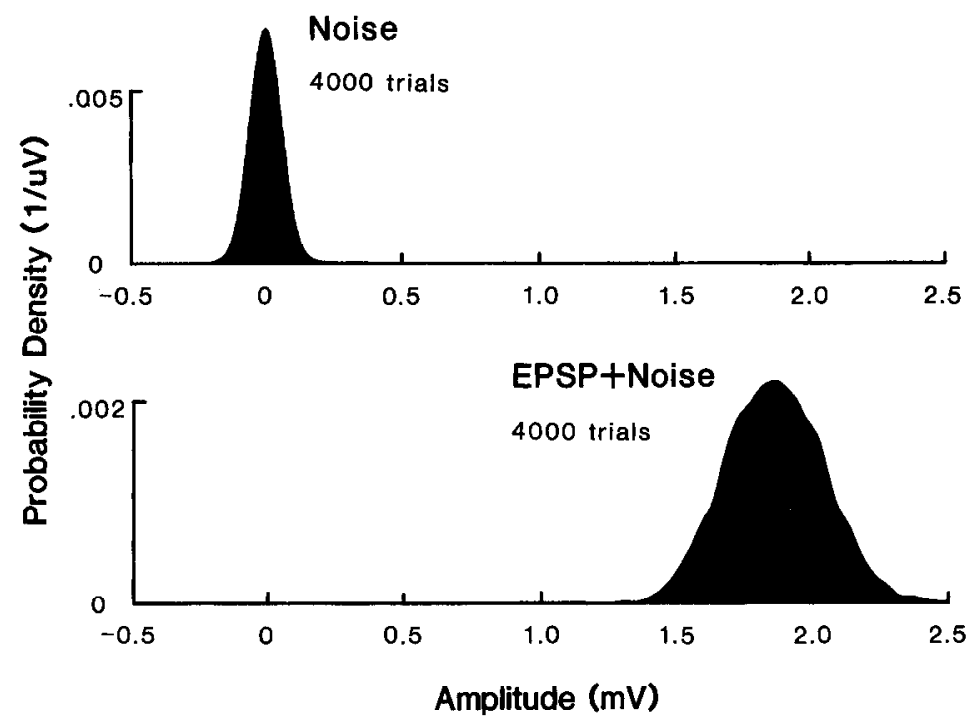

Nerves in the left hindlimb to posterior biceps, semitendinosus, medial gastrocnemius, lateral gastrocnemius-soleus, and plantaris were dissected free and cut at their entry to the muscle. The nerves were placed on bipolar stimulating electrodes. The lumbosacral cord was exposed by laminectomy from L2 to L7. Dorsal roots L 7 and S1 were separated into thin filaments left in continuity. These filaments were placed sequentially on a recording electrode and the response examined to stimulation of each of the exposed hindlimb nerves. A filament was selected if single group I fibers could be stimulated from one or more of the hindlimb nerves. Following selection of a suitable dorsal root filament, all other dorsal roots from L6 to S2 were cut. All ventral roots from L6 to $\mathrm{S} 2$ were also cut.

Figure 1 illustrates the stimulation and recording procedure. Identification of DSCT neurons was performed by antidromic stimulation of the dissected dorsolateral fasciculus of the spinal cord at the C2 level (Houchin et al., 1983; Tracey and Walmsley, 1984). The dorsal columns were removed for approximately $2 \mathrm{~cm}$ to prevent stimulation spreading to them. Intracellular recordings were made from DSCT neurons at the level of L3 and L4 dorsal root entry zones. The electrode, filled with 3 м $\mathrm{KCl}$ or $2 \mathrm{M} \mathrm{K}$-methyl sulfate was driven into the spinal cord approximately $200-300 \mu \mathrm{m}$ from the midline. Single fiber EPSPs were evoked by stimulating the muscle nerve and were recorded onto computer disk for subsequent analysis.

\section{Data analysis}

For each single fiber EPSP, measurements were made of the peak amplitudes and a histogram of these amplitudes constructed. Between 800 and 4600 samples were used in the construction of each histogram. A baseline and peak measurement region were selected for each EPSP. These regions were chosen to maximize the ratio of signal standard deviation to noise standard deviation. This was designed to improve the resolution of the subsequent fluctuation analysis. The same measurement regions were applied to the noise-alone records and a histogram of noise amplitudes constructed. In practice, smoothed frequency polygons were used (Fig. 1), as these preserved more information and 
avoided problems associated with the choice of bin widths in histograms (see Clements et al., 1986). The smoothed frequency polygons were normalized by the population sample to obtain the probability density curves illustrated.

\section{EPSP fluctuation analysis}

Two different methods were applied to each single fiber EPSP. Both methods assume statistical independence of the noise and EPSP amplitude fluctuations.

Deconvolution analysis. A numerical deconvolution procedure was used to determine the EPSP amplitude fluctuation pattern. Briefly, the method is as follows: Distributions of noise amplitudes and noise-contaminated EPSP amplitudes are constructed. The problem is to determine the noise-free fluctuation pattern in EPSP amplitude. An optimization procedure is used to find a noise-free fluctuation pattern that, when "contaminated" or convolved with the measured noise distribution, gives the best fit to the measured (i.e., noise-contaminated) EPSP distribution. Previous deconvolution procedures have used the leastsquared error (LSE) optimization procedure (Edwards et al., 1976; Wong and Redman, 1980; Jack et al., 1981; Redman and Walmsley, 1983; Tracey and Walmsley, 1984). However, Ling and Tolhurst (1983) have shown that the maximum likelihood estimator (MI.F) approach generally produces much more reliable results. This procedure finds the values of the underlying component amplitudes and associated probabilities that maximize the likelihood of observing the measured data, i.e., the EPSP probability density distribution. In the present study we have used both the LSE and MLE deconvolution procedures. The MLE method used in the present study was developed by Clements et al. (1986) and is based on procedures described by Clayton and Ulrych (1977) and Ling and Tolhurst (1983).

An important aspect of the deconvolution procedure is that no a priori assumptions are made about the component amplitudes and probabilities of the underlying fluctuations.

Simple binomial model. An optimization procedure was developed in which, for a given population $N$, the optimum quantal size $(d V)$ and probability $(P)$ are determined. The optimization was such that the binomial probability distribution, convolved with the measured noise distribution, gave the best fit to the measured (i.e., noise-contaminated) EPSP amplitude distribution. The value of $N$ was varied from 1 to over 100 , and the binomial solutions for $d V$ and $P$ for each $N$ were examined and compared (see Results). Optimal values of $P$ and $d V$ were found using both the LSE and MLE approaches with a multidimensional simplex procedure (Kowalik and Osborne, 1968, Chap. 2.6). Initially, the solution obtained from this procedure was cross-checked by systematically varying the parameters $P$ and $d V$, for a given $N$, over a wide range of values to verify that a true optimum had been obtained.

An important assumption underlying the binomial analysis presented in this paper is that the quantal event has a variance that is insignificant compared to the noise. This assumption is based on experimental evidence at this synapse (Tracey and Walmsley, 1984; Walmsley et al., 1986) and at the Ia fiber-motoneuron connection (Edwards et al., 1976; Jack et al., 1981).

As an additional basis for comparison of the fits obtained using the deconvolution and binomial procedures, $\chi^{2}$ values were calculated for all results. Class intervals were identical for both the deconvolution and binomial fits, and for all class intervals the expected frequency was $>5$. The number of degrees of freedom $(d f)$ in the $\chi^{2}$ test is equal to the number of class intervals minus one, minus the number of parameters of the hypothesized distribution estimated by sample statistics (Hines and Montgomery, 1972). In the case of the binomial model there are 3 such parameters $(N, P$, and $d V)$. In the case of the deconvolution result, the number of parameters is equal to twice the number of components minus one. [The amplitudes of these components and all but one (since the sum of probabilities must be unity) of the probabilities of these components can be adjusted independently to obtain a fit.]

\section{Results}

Intracellular recordings were obtained from DSCT neurons in 43 adult cats. Single group I fiber EPSPs were selected for analysis if the membrane potential of the neuron was stable and more negative than $-65 \mathrm{mV}$, the average peak amplitude of the EPSP did not drift during the recording period, and the background noise level was sufficiently low (usually $<100 \mu \mathrm{V}$ rms). Thirteen EPSPs satisfied these requirements, and the results of the analysis of these EPSPs are presented.

\section{Deconvolution analysis}

Figure 2 presents deconvolution results from 2 different single group I fiber EPSPs. Figure 2, $A$ and $E$, shows the measured probability density curves of the noise, and the EPSP + noise, for each EPSP. The signal-to-noise ratio for these 2 examples was large enough to allow individual peaks and regions of inflection to be observed on the measured EPSP + noise probability density distributions. The underlying fluctuation patterns for each EPSP were determined using the numerical deconvolution procedure described in Materials and Methods. Results using both LSE and MLE optimizations were compared for all EPSPs. Figure 2, $B$ and $C$ (EPSP 1), and 2, $F$ and $G$ (EPSP 2), illustrates the results using MLE optimization. The unbroken curves in Figure 2, $B$ and $F$, are the measured EPSP + noise distributions, and the overlying dotted curve is the proposed fit using the MLE deconvolution procedure. Both EPSP 1 and EPSP 2 were found to fluctuate between 6 discrete amplitudes, as illustrated in Figure 2, $C$ and $D$, respectively. The individual components, with appropriate noise added are shown as individual dotted curves in Figure $2, B$ and $F$. (The overlying dotted curve is the sum of these component curves.) Figure 2, $D$ and $H$, illustrates the results using the LSE deconvolution procedure for comparison. The solution for the LSE procedure gave 6 discrete fuctuation components for each EPSP, and the form of the solution was very similar to the MLE results in each case. The reconvolved fits (dotted curves in Fig. 2, $D$ and $H$ ) were also very good: the $\chi^{2}$ values for EPSP 1 are $4.2(65 d f)$ for the MLE deconvolution fit and $9.8(65 d f)$ for the LSE deconvolution fit. Corresponding $\chi^{2}$ values for EPSP 2 are $1.5(50 d f)$ for the MLE and $1.3(50 d f)$ for the LSE deconvolution fits, respectively. From these values, all fits cannot be rejected at the $0.05,0.01$, and 0.001 levels of significance. [Although the solutions obtained using the MLE and LSE deconvolution procedures produced similar $\chi^{2}$ values, only MLE results are presented for the remaining examples since this method has been shown to be more reliable (Ling and Tolhurst, 1983).]

The 2 examples shown in Figure 2 are notable because they exhibit individual peaks and inflections in the measured EPSP + noise probability density distributions. Such peaks are readily observed in the probability distributions of end-plate potentials at the neuromuscular junction, where the signal-to-noise ratio is considerably better than at mammalian central synapses (del Castillo and Katz, 1954). To demonstrate that these peaks and inflections were consistent features, the population of EPSPs making up the distribution of EPSP 2 (Fig. 2) was divided into halves, and each subpopulation was also found to exhibit these pcaks and inflections. The MLE deconvolution procedure was applied to the total population and to each of the 2 subpopulations. In all 3 cases, 6 discrete components were obtained, with slight variations in probabilities and locations (due to sampling errors) but nevertheless correlating well with the peaks and inflections in the probability density distributions.

It should be emphasized that the MLE deconvolution procedure generally gave discrete components that correlated well with these inflections, even though it was not constrained to do so (see Fig. 6).

Figure 3 presents the fluctuation components for all 13 single fiber EPSPs, arranged in order of increasing average amplitude 

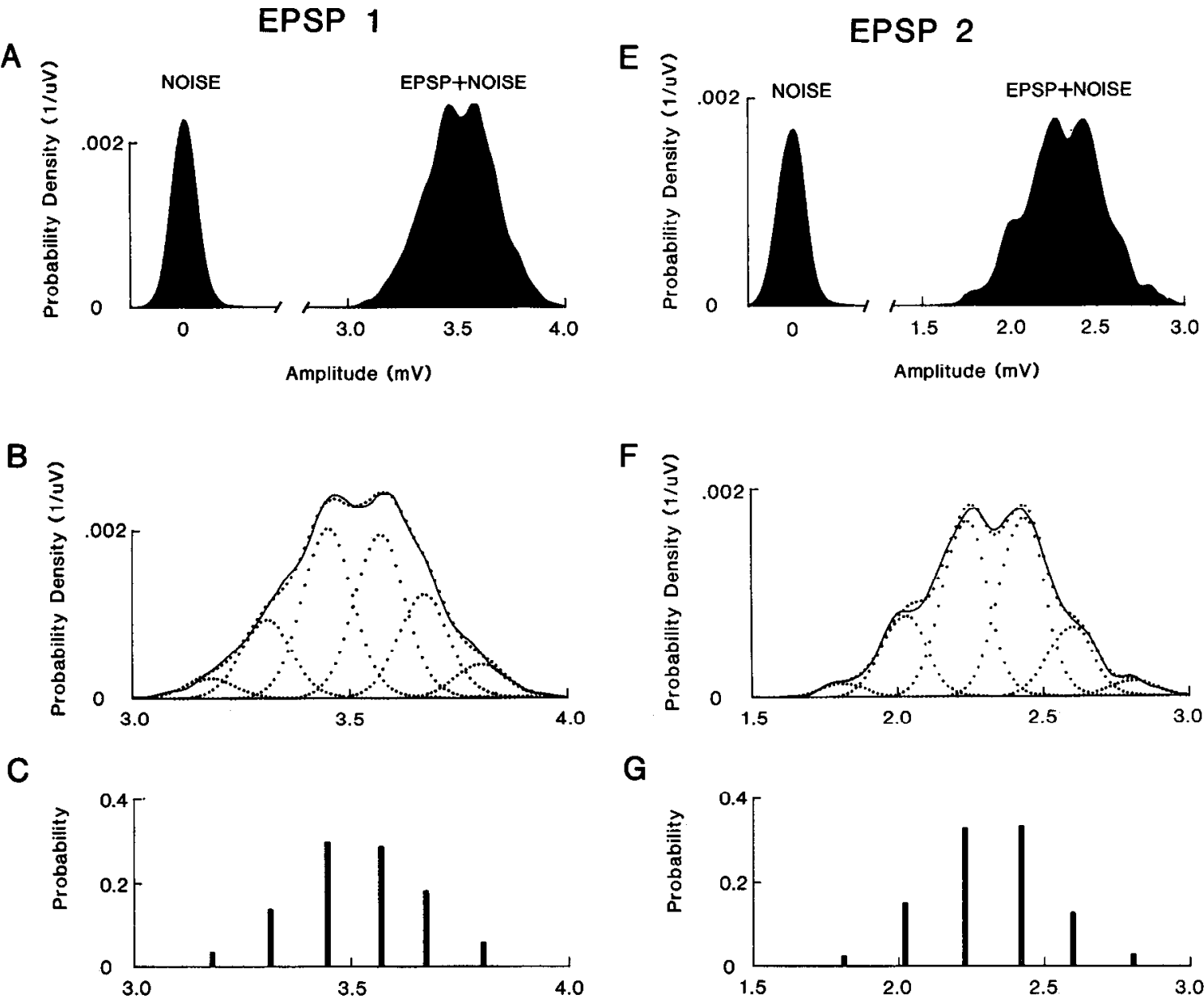

G
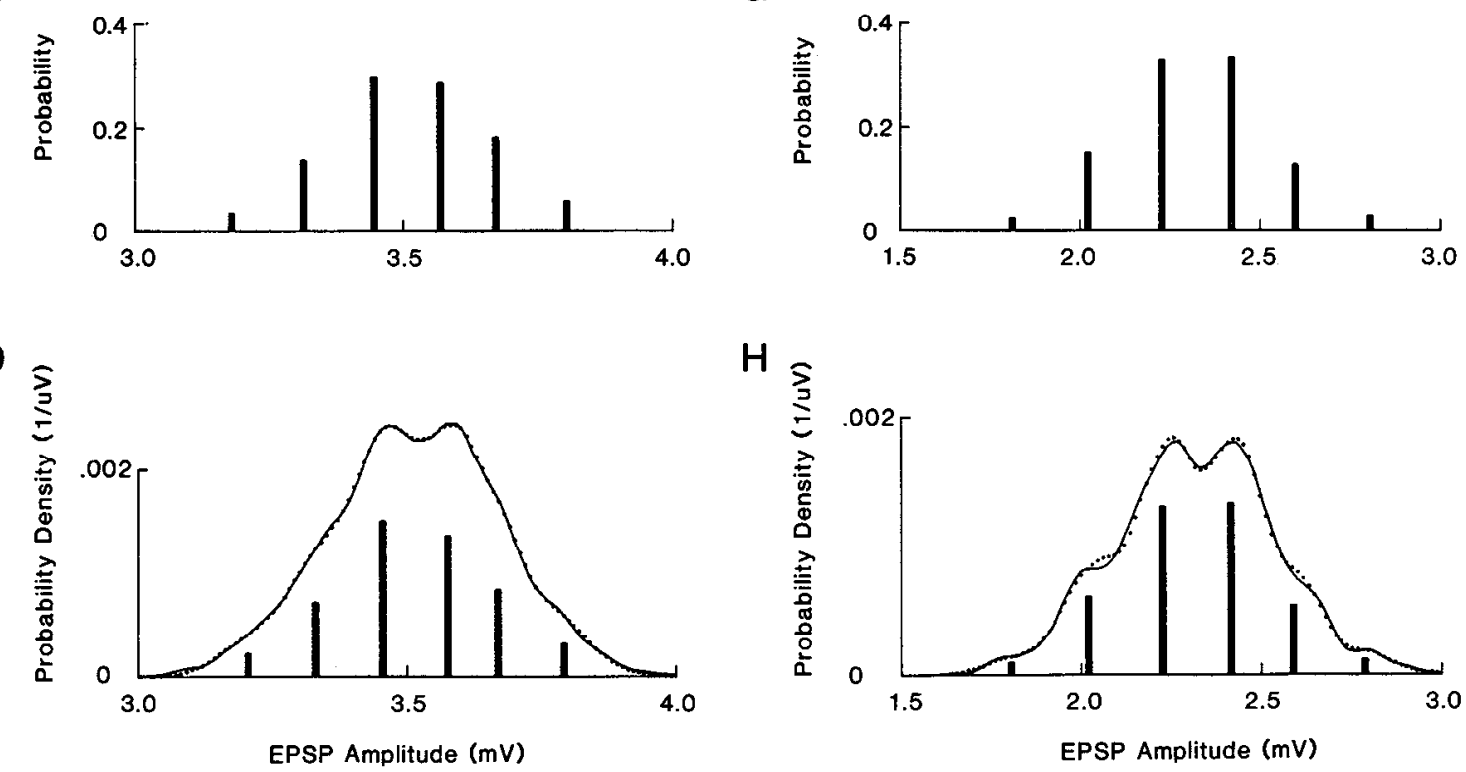

Figure 2. Deconvolution fluctuation analysis of 2 diffcrent single-fibcr EPSPs. $A-D$, Results for EPSP 1 (2124 trials). $E-H$, Corresponding results for EPSP 2 (900 trials). EPSPs 1 and 2 correspond to EPSPs M and L shown in Figure 4 and Table 1. $A$ and $E$, Probability density distributions for evoked EPSP (EPSP + noise) and noise. Note: Noise distributions have been scaled down by a factor of $3 . B$ and $C, F$ and $G$, Results of fluctuation analysis using MLE deconvolution procedure. $D$ and $H$, Results of fluctuation analysis using LSE deconvolution procedure. Solid curves in $B, D, F$, and $H$ are the measured EPSP probability density distributions. The overlying dotted curves are the fits obtained using the deconvolution procedures. Solid bars in $C$ and $G$ are the EPSP fluctuation components (noise free) obtained from the MLE deconvolution. Solid bars in $D$ and $H$ (same probability scale as $C$ and $G$ ) are the component probabilities obtained from the LSE deconvolution. Note nonzero amplitude axis origins.

$(A-M)$. In general, these EPSPs fluctuated between discrete amplitudes with approximately equal increments between components. The average increment for each EPSP varied from 91 to $198 \mu \mathrm{V}$, and the average value for all EPSPs was $144 \mu \mathrm{V}$. The number of discrete components varied from 2 to 6 . None of the EPSPs exhibited failures of response. No unifying pattern was obvious from these results, although there was a tendency for larger EPSPs to exhibit more components. The $\chi^{2}$ goodnessof-fit tests (Table 1) showed that the results obtained using the deconvolution procedure gave very good fits to the measured data. In all cases these fits could not be rejected at the 0.05 , 0.01 , and 0.001 levels of significance.

\section{Binomial analysis}

Three parameters are required for a simple binomial model. In the context of synaptic potentials, these are the population size, $N$, the unit or quantal amplitude, $d V$, and the quantal release probability, $P$. The simple binomial model is based on all quan- 


\section{MLE Deconvolution Results}
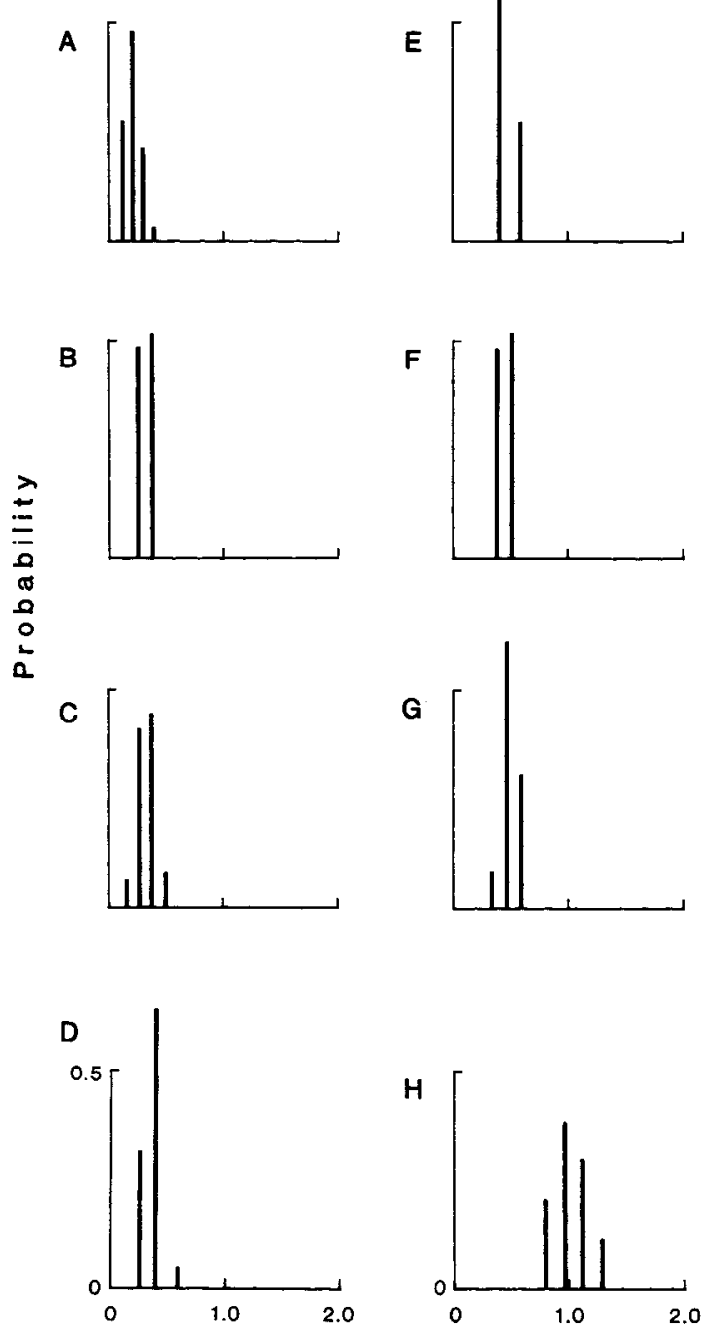

EPSP Amplitude ( $m V)$
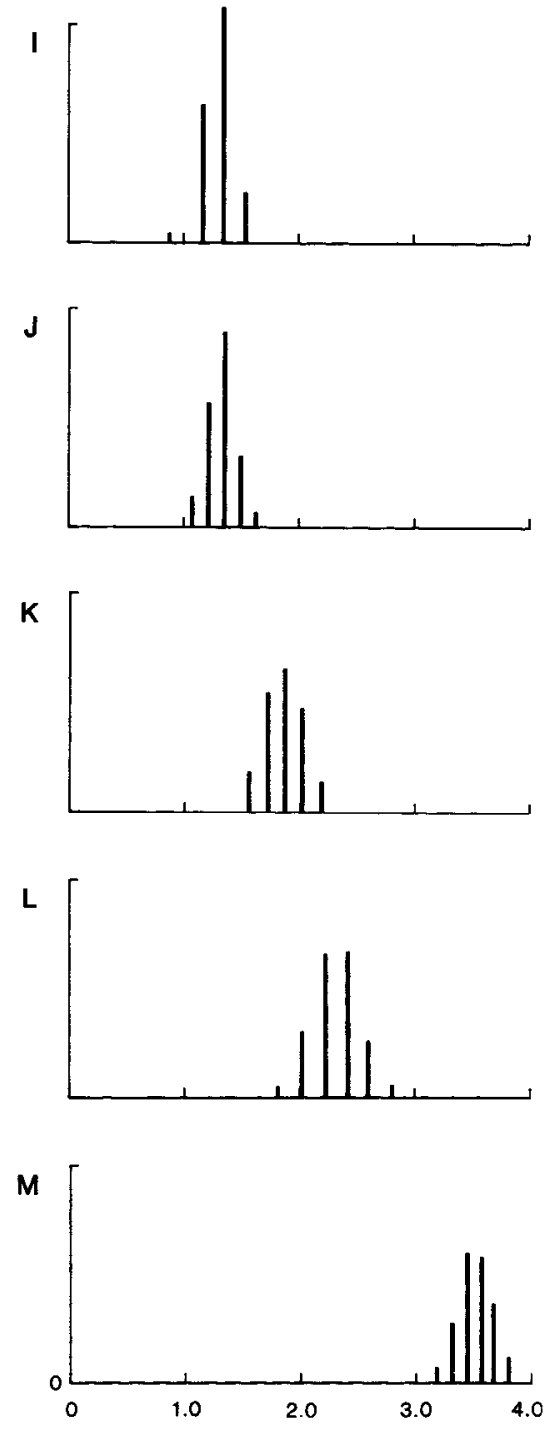

$\mathbf{L}$

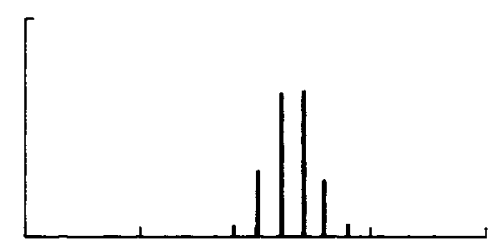

Figure 3. Fluctuation components determined by the MLE deconvolution procedure for 13 different single group I fiber EPSPs recorded in DSCT neurons. Results arranged in order of increasing average peak amplitude from $A$ to $M$ (corresponding to $A-M$ in Table $1)$. tal events having the same probability of occurrence. At the neuromuscular junction, at least one of these parameters, the quantal size, can be measured. At a central neuron, it is not possible to determine any of these parameters a priori. Therefore, a procedure was used in which it was not necessary to make any assumptions about the binomial parameters. The population size, $N$, was varied from 1 to $>100$, so that the form of the solution could be examined and compared for a large range of values of $N$. For a given $N$, the problem is reduced to finding the quantal parameters $d V$ and $P$. An optimization procedure was used to find the values of $d V$ and $P$ that gave the best fit to the data, for each $N$. It was possible, of course, to vary all 3 parameters $-N, d V$, and $P$ - to obtain an overall optimal solution. However, as shown below, it is important to examine the form of the solution for different values of $N$. (Since $N$ is an integer variable, the overall optimal solution can easily be found by inspection of the goodness-of-fit values over the whole range of values of $N$.)

The simple binomial solutions for one EPSP (the same EPSP as EPSP 2 in Fig. 2) are illustrated in Figure 4 for several values of $N(=12,14,16$, and 40$)$. At the right of the figure are shown the component probabilities for the simple binomial solution for each $N$. The reconvolved curve is shown at the left (dotted curve) and compared with the measured EPSP + noise distribution (unbroken line). The $\chi^{2}$ statistic was examined for each value of $N$. Inspection of this value showed that the overall best fit was obtained for $N=40$. However, this solution produced a smooth, Gaussian-like envelope that did not reproduce any of the peaks and inflections seen in the measured EPSP + noise distribution. Since it had already been demonstrated that such inflections were a consistent and significant feature of the EPSP + noise distributions, it was considered important to include these as an additional criterion in selecting the most appropriate binomial solution for comparison. On this basis, the smooth curve generated by $N=40$ in Figure 4 is an inappropriate solution. The reason that the $N=40$ solution produces a better $\chi^{2}$ statistic in this case becomes obvious if the form of the solution for values of $N<40$ is examined. Inspection of the reconvolved 
Simple Binomial
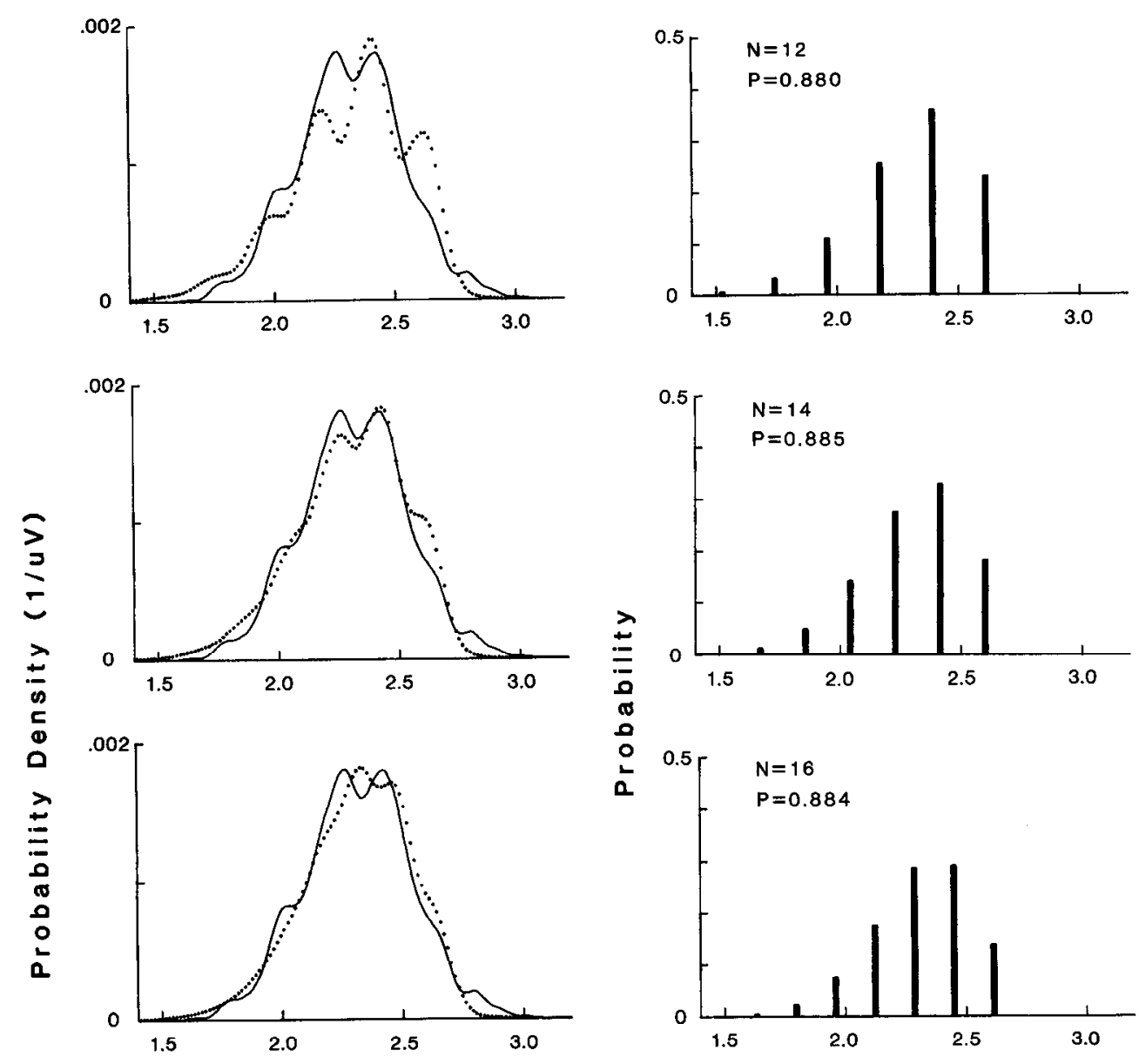

Figure 4. Results of simple binomial fits for 4 different values of $N(12,14$, 16 , and 40 ) to the probability density distribution for a single-fiber EPSP (same as EPSP 2 in Fig. 2). Solid curves, Measured EPSP probability density distribution; dotted curves, simple binomial fit. Binomial probability components for each value of $N$ shown at right. Note nonzero amplitude axis origins.

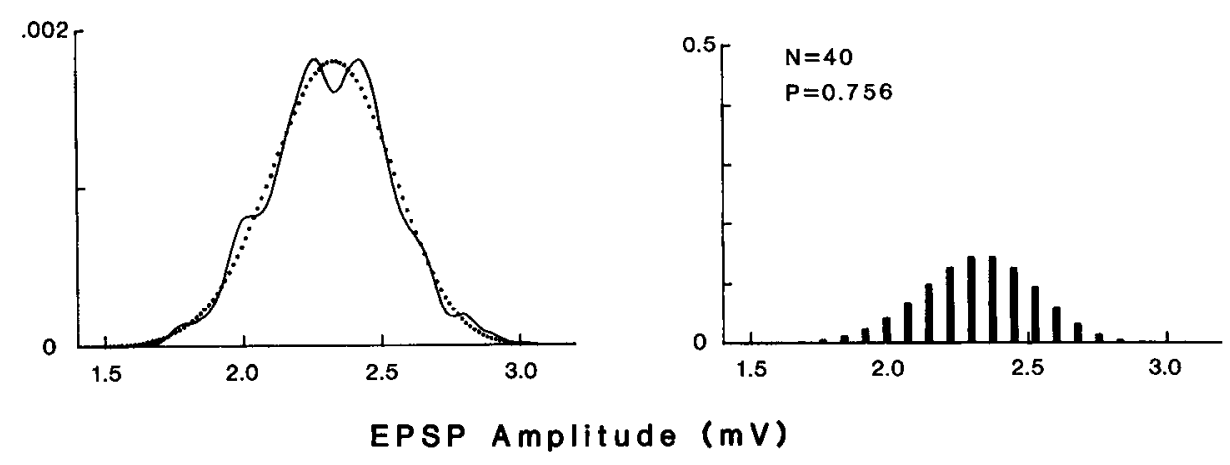

distributions for a range of values of $N$ revealed that an $N$ equal to 14 (Fig. 4) produced a yuantal increment approximately matching the peaks and inflections of the measured distribution. Values of $N<14$ (e.g., $N=12$; Fig. 4) and values of $N>14$ (e.g., $N=16$; Fig. 4) produced peaks that did not align with the measured curve. However, examination of the form of the fit between the binomial model and the measured data curves for $N=14$ shows that there is a gross mismatch, particularly in the tails of the distributions (dotted and continuous curves in Fig. $4, N=14$ ). This provides an explanation for the better $\chi^{2}$ statistic for $N=40$, where the binomial and measured curves are more closely matched in the tails of the distributions. The point should be made that if the underlying distribution is truly not binomial, then the overall best fit to a binomial ( $N=40$ in this case) is meaningless.
An obvious feature of the binomial solution in this example was that as $N$ was increased above 14 (e.g., to $N=16$; Fig. 4), the quantal increment did not remain at the same, apparently appropriate, spacing but decreased. This decrease then caused the peaks and inflections to become misaligned. Subjectively, it might seem that the binomial fit could be improved by keeping the quantal increment the same as $N=14$ and to simply add an additional component, since it appears that the lack of such a component is causing a mismatch in the upper tail of the $N=$ 14 solution (Fig. 4). Figure 5 illustrates the results of investigating this suggestion and reveals some of the constraints of the simple binomial model. Figure $5 A$ shows the optimal simple binomial solution for $N=14$ (as in Fig. 4). The binomial probability in this case is $P=0.885$. Figure $5, D$ and $E$, illustrates the effect of keeping the quantal increment the same (as $N=$ 

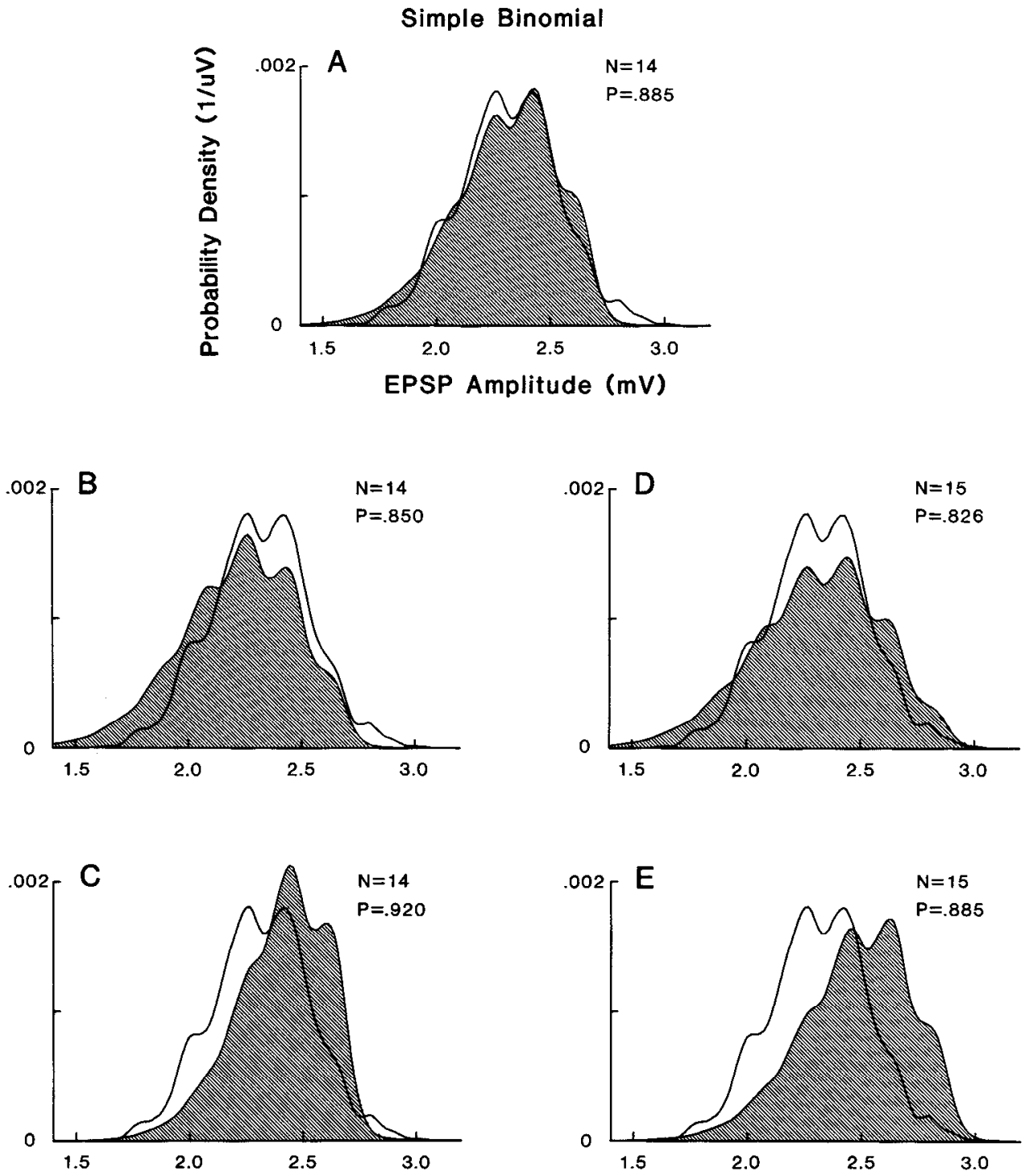

Figure 5. Effect of altering the binomial parameters $N$ and $P$ on the form of the simple binomial fit (shaded distribution) to the measured EPSP probability density distribution (solid curve) for a single-fiber EPSP (same as Fig. 5). In all cases, the quantal amplitude is $186.6 \mu \mathrm{V}$. Note nonzero amplitude axis origins.
14) but adding a further component, as suggested above, to obtain a binomial fit for $N=15$. Figure $5 E$ illustrates the solution if an extra component is simply added, keeping $P$ the same as for the $N=14$ solution $(P=0.885)$. Inspection of the fit between the binomial and the measured distribution indicates an obvious mismatch, with the binomial curve apparently shifted to the right. Figure $5 D$ illustrates the optimal fit (at the same quantal spacing as $N=14$ ) for $N=15$, which resulted in a reduction of $P$ to 0.826 . This represents the best binomial fit to the measured distribution, incorporating an alignment of the peaks, and including the extra component apparently lacking in the solution for $N=14$. Even so, there is a considerable mismatch between the binomial curve and the measured EPSP + noise distribution. The binomial distribution predicts much larger probabilities in both tails of the distribution and too little in the central region. Given that, in this case, $N$ and $d V$ are appropriate, the inherent constrained nature of the simple binomial does not allow the peakedness of the distribution to be increased so as to align it with the measured distribution. Furthermore, adjustment of $P$ simply results in skewness to the left or right, causing a worse fit. [Figure $5, B$ and $C$, demonstrates the effect of keeping $N(14)$ and $d V$ constant, and reducing $(B)$ or increasing $(C)$ the probability, $P$, slightly. Such slight changes result in obvious mismatches, which are also reflected in the $\chi^{2}$ values. When $P$ is reduced (Fig. $5 B$ ), the bionomial distribution becomes more symmetrical, but this results in a large mismatch, especially in the lower tail of the distribution. If $P$ is increased (Fig. 5C), the distribution becomes narrower and apparently shifts to the right, causing mismatches of both the lower and upper tails of the distribution.] Increasing $N$ to greater than 15 results in an even worse fit because the spread of the binomial distribution is further increased. In the example given above (Figs. 4 and 5), all attempts to adjust $N, P$, and $d V$ to obtain a good simple binomial fit failed. The best fit consistent with the coincidence and alignment of peaks in the measured EPSP + noise distribution was obtained with $N=15, P=0.826$, and $d V=186.6 \mu \mathrm{V}$. The $\chi^{2}$ test rejected this binomial fit at the 0.05 , 0.01 and 0.001 significance levels.

All 13 single-fiber EPSPs were analyzed using the same procedure outlined above. Figure 6 illustrates a comparison among 4 solutions obtained by the MLE deconvolution and the optimal simple binomial method (including the previous example, Fig. $6 B$ ). In each case, the binomial solution illustrated has been chosen according to the same criteria applied to the example 

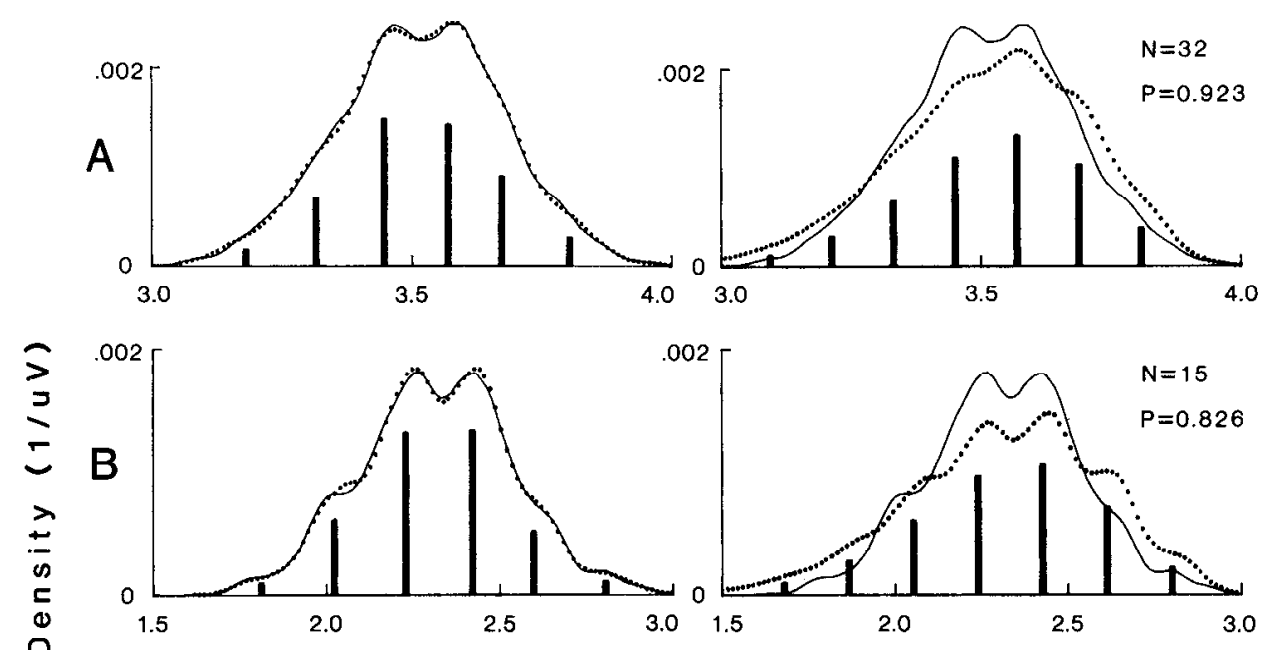

Figure 6. Results of amplitude fluctuation analysis for 4 different singlefiber EPSPs (arranged in order of decreasing amplitude, $A-D$ ). Solid curves are the measured EPSP probability density distributions and the dotted curves are the fits for the MLE deconvolution procedure (left) or the simple binomial model (right). The underlying fluctuation components are shown as solid bars in each case. These components have been scaled appropriately for cach plot: probability scalcs for $A, B$, $C$, and $D$ are $0.4,0.5,1.0$, and 0.75 , respectively. Note the nonzero amplitude axis origins.
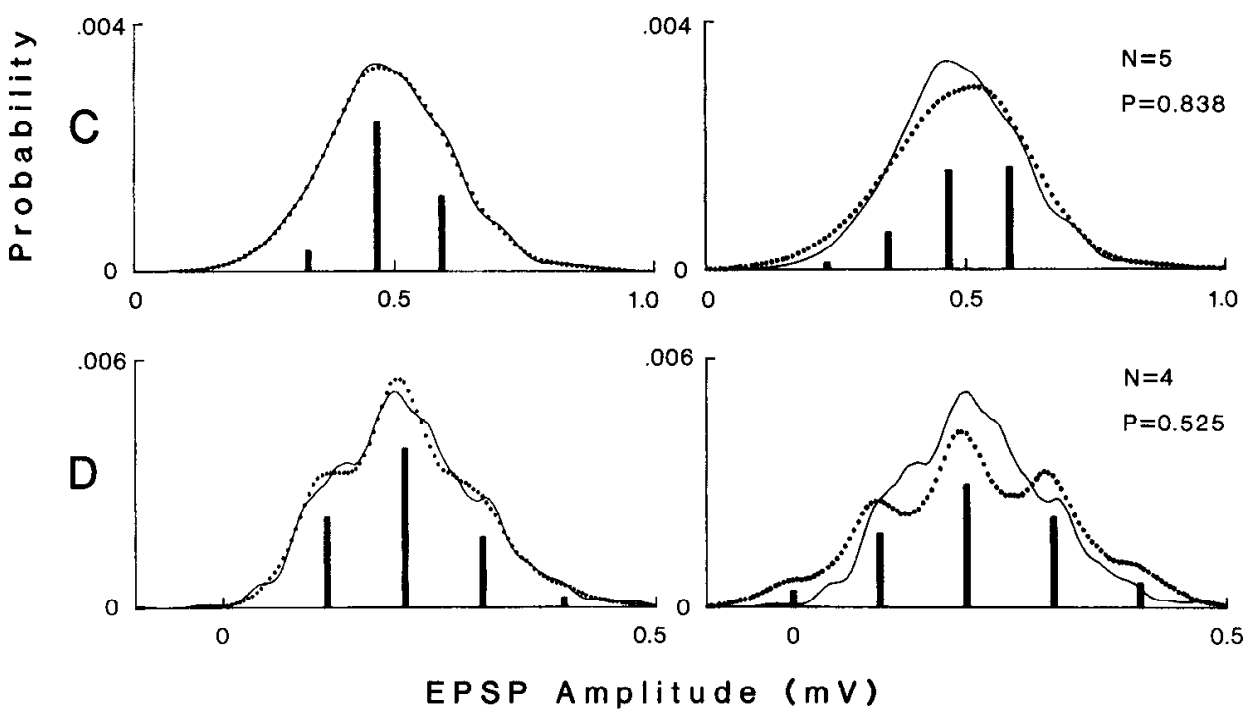

given in Figures 4 and 5. The MLE deconvolution results provide good fits to the measured data (see Table 1). In contrast, the simple binomial distributions all demonstrate gross mismatches with the measured EPSP + noise distributions.

Table 1 provides a comparison of the results obtained using the MLE deconvolution procedure and the simple binomial model. Note that the binomial distributions used in this comparison have been chosen according to the criteria outlined in the discussion of the example shown in Figures 4 and 5. From the $\chi^{2}$ values it is evident that the solutions obtained using the MLE deconvolution procedures give much better fits to the measured data than the simple binomial model in all cases. Application of the $\chi^{2}$ goodness-of-fit test showed that 12 of 13 binomial fits could be rejected at the 0.05 and 0.01 levels of significance and 11 of 13 could be rejected at the 0.001 significance level. By comparison, none of the MLE deconvolution fits could be rejected at these significance levels.

\section{Discussion}

In the mammalian CNS, the most studied synaptic connection is that between single Ia afferent fibers and spinal motoneurons. Early attempts to fit Poisson or binomial models to fluctuations in the amplitude of single Ia fiber EPSPs were not successful, but this was attributed to factors such as nonlinear summation of synaptic potentials (Kuno, 1964; Kuno and Miyahara, 1969). Mendell and Weiner (1976) analyzed the fluctuations in single Ia fiber EPSPs in motoneurons and fitted their results to either Poisson or binomial models, although some results could not be described by either model. More recently, Jack et al. (1981) found, using deconvolution analysis, that the fluctuations of Ia EPSPs in motoneurons could not be described by binomial or Poisson statistics. It is generally agreed, however, that synaptic transmission at the Ia fiber-motoneuron connection occurs in a quantal manner. In addition, it has been proposed that the quantal unit is the result of all-or-nothing transmission at a synaptic bouton and that the overall EPSP fluctuation pattern is the sum of such transmission at all synaptic boutons in the connection (Edwards et al., 1976; Jack et al., 1981; Redman and Walmsley, 1981, 1983).

The present study has examined excitatory synaptic transmission between single group I afferents and DSCT neurons in the cat spinal cord. In agrecment with a previous study (Tracey and Walmsley, 1984), single group I fiber EPSPs were found to fluctuate between discrete amplitudes, separated by equal, or quantal, increments. Supporting evidence for discrete components, rather than a continuous distribution, was indicated in 
Table 1. Comparison of the fits between the measured EPSP probability distributions and the distribution obtained using the deconvolution procedure or the simple binomial model

\begin{tabular}{|c|c|c|c|c|c|c|c|c|c|c|c|c|c|c|}
\hline \multirow[b]{4}{*}{ EPSP } & \multirow{4}{*}{$\begin{array}{l}\text { Average } \\
\text { peak } \\
\text { ampli- } \\
\text { tude } \\
(\mu \mathrm{V})\end{array}$} & \multirow{4}{*}{$\begin{array}{l}\text { No. of } \\
\text { samples }\end{array}$} & \multicolumn{5}{|c|}{ Deconvolution fits } & \multirow{2}{*}{\multicolumn{7}{|c|}{ Simple binomial fits }} \\
\hline & & & \multirow{3}{*}{$\begin{array}{l}\text { Average } \\
\text { com- } \\
\text { ponent } \\
\text { ampli- } \\
\text { tude } \\
(\mu \mathrm{V})\end{array}$} & \multirow{3}{*}{$\begin{array}{l}\chi^{2} \text { value } \\
(d f) \\
\end{array}$} & \multirow{2}{*}{\multicolumn{3}{|c|}{ Significance levels }} & & & & & & & \\
\hline & & & & & & & & \multirow[b]{2}{*}{$d V$} & \multirow[b]{2}{*}{$N$} & \multirow[b]{2}{*}{$P$} & \multirow{2}{*}{$\begin{array}{l}\chi^{2} \text { value } \\
(d f)\end{array}$} & \multicolumn{3}{|c|}{ Significance levels } \\
\hline & & & & & 0.05 & 0.01 & 0.001 & & & & & 0.05 & 0.01 & 0.001 \\
\hline A & 210 & 2400 & 91 & $25.3(75)$ & $*$ & $*$ & $*$ & 100 & 4 & 0.53 & $870.4(79)$ & - & - & - \\
\hline B & 320 & 1590 & 125 & $28.8(89)$ & $*$ & $*$ & $*$ & 127 & 3 & 0.84 & $122.8(89)$ & - & - & $*$ \\
\hline $\mathrm{C}$ & 330 & 3600 & 115 & $9.2(51)$ & * & $*$ & * & 92 & 5 & 0.72 & $160.3(55)$ & - & - & - \\
\hline D & 357 & 875 & 167 & $8.1(57)$ & $*$ & $*$ & * & 130 & 5 & 0.55 & $317.2(59)$ & - & - & - \\
\hline $\mathrm{E}$ & 454 & 4200 & 184 & $61.2(110)$ & $*$ & * & * & 200 & 3 & 0.76 & $1531.2(110)$ & - & - & - \\
\hline F & 459 & 800 & 132 & $13.8(51)$ & $*$ & $*$ & * & 123 & 4 & 0.88 & $32.0(51)$ & $*$ & $*$ & $*$ \\
\hline $\mathrm{G}$ & 490 & 3100 & 129 & $16.2(64)$ & $*$ & $*$ & $*$ & 117 & 5 & 0.84 & $163.8(66)$ & - & - & - \\
\hline $\mathbf{H}$ & 1017 & 4600 & 165 & $37.4(124)$ & * & $*$ & $*$ & 160 & 8 & 0.79 & $185.7(128)$ & - & - & - \\
\hline I & 1299 & 860 & 186 & $7.0(62)$ & $*$ & $*$ & $*$ & 193 & 8 & 0.84 & $183.1(66)$ & - & - & - \\
\hline J & 1326 & 1720 & 138 & $6.2(78)$ & $*$ & $*$ & $*$ & 135 & 12 & 0.82 & $525.0(84)$ & - & - & - \\
\hline $\mathrm{K}$ & 1861 & 4000 & 118 & $22.2(84)$ & $*$ & $*$ & $*$ & 124 & 18 & 0.83 & $598.7(91)$ & - & - & - \\
\hline $\mathrm{L}$ & 2315 & 900 & 198 & $1.3(50)$ & $*$ & $*$ & $*$ & 187 & 15 & 0.83 & $225.7(58)$ & - & - & - \\
\hline M & 3516 & 2124 & 125 & $4.2(65)$ & $*$ & $*$ & $*$ & 119 & 32 & 0.92 & $306.2(73)$ & - & - & - \\
\hline
\end{tabular}

In each case, acceptance at a level of significance in the $\chi^{2}$ goodness-of-fit test is indicated by an asterisk. EPSPs A-M correspond to those shown in Figure 3.

the present study by the existence of consistent peaks and regions of inflection in the measured EPSP probability density distributions. The deconvolution procedure gave probability distributions that closely fitted the observed data and produced EPSP amplitude components that closely aligned with these peaks and inflections. Rather than assume that the deconvolution results were correct and attempt a binomial fit to these components (e.g., Jack et al., 1981), an independent procedure was employed that made no a priori assumptions about the binomial parameters $N, P$, or $d V$. Even so, it was not possible to obtain a satisfactory simple binomial fit in almost every case. [It is also worth emphasizing that considerable caution should be exercised in accepting a simple binomial solution on the basis of goodncss-of-fit critcria alonc, as clcarly illustrated by Barton and Cohen (1977) and Brown et al. (1976).]

Since the binomial model is apparently not appropriate at this synapse, what information can be obtained from the deconvolution results, which do provide an adequate description? The deconvolution results demonstrate that synaptic transmission at this connection occurs in a probabilistic manner, in which the EPSP fluctuates between discrete amplitudes separated by approximately equal increments. However, it is not possible to interpret the magnitudes of the probabilities of these discrete levels without making some assumptions about the underlying events. In the binomial model, the assumptions are that there is a stationary population, $N$, composed of quantal units, each having an identical probability of occurrence. Since the simple binomial model does not apply, then 1 or more of these assumptions must be incorrect.

The anatomical details of the connection between single group I afferents and DSCT neurons have been revealed in previous light- and electron-microscopic studies (Tracey and Walmsley, 1984; Walmsley et al., 1985). These studies showed that a single fiber gives rise to a variable number of boutons making connection with a DSCT neuron. These boutons may vary greatly in size, from $1 \times 1 \mu \mathrm{m}$ to $20 \times 3 \mu \mathrm{m}$. In addition, a single bouton may contain a variable number of synaptic specializations, or transmitter release sites. In a recent electron-microscopic study (Walmsley et al., 1987) it was also shown that some, but not all, group Ia and Ib boutons in Clarke's column receive presynaptic contacts. Such contacts are presumably related to presynaptic inhibition of transmission at these terminals. Presynaptic inhibition at some boutons and not others, arising from the same afferent fiber, could produce large differences in release probabilities between boutons.

A number of previous studies on synaptic transmission in the CNS have attempted to relate fluctuations in EPSP amplitude with the number of synaptic boutons (Edwards et al., 1976; Jack et al., 1981; Korn et al., 1981; Redman and Walmsley, 1981, 1983). Tracey and Walmsley (1984) proposed that transmission between group I muscle afferents and DSCT neurons occurs with EPSP fluctuations related to transmitter release sites, rather than boutons per se, since many boutons contain multiple transmitter release sites. There are now a number of ultrastructural studies on identified terminations in the spinal cord and brain stem that give much wider support for this proposal. Single boutons containing multiple synaptic specializations have been described for Ia terminations in the ventral horn (Fyffe and Light, 1984) and cutaneous mechanoreceptive and hair follicle afferent boutons in the lumbosacral spinal cord (Maxwell et al., 1982, 1984; Bannatyne et al., 1984; Semba et al., 1984). Many of these boutons were also found to be contacted by presynaptic terminals.

Tracey and Walmsley (1984) suggested that the probability of transmitter release may vary from release site to release site. Variation in release probability may occur from bouton to bouton, or possibly from release site to release site within the same bouton. Such a nonuniform release probability could explain the failure of the simple binomial model in the present study and at other synapses (see also Barton and Cohen, 1977; Bennett and Lavidis, 1979; Jack et al., 1981; d'Alonzo and Grinnell, 1985). We believe that further study of the group I afferent- 
DSCT neuron connection will provide a useful and general model of excitatory synaptic transmission in the mammalian CNS. We are presently exploring a number of alternative models in an attempt to gain further insight into the mechanisms of synaptic transmission at this connection.

\section{References}

Bannatyne, B. A., D. J. Maxwell, R. E. W. Fyffe, and A. G. Brown (1984) Fine structure of primary afferent axon terminals of slowly adapting cutaneous receptors in the cat. Q. J. Exp. Phys. 69: 547557.

Barton, S. B., and I. S. Cohen (1977) Are transmitter release statistics meaningful? Nature 268: 267-268.

Bennett, M. R., and N. A. Lavidis (1979) The effect of calcium ions on the secretion of quanta evoked by an impulse at nerve terminal release sites. J. Gen. Physiol. 74: 429-456.

Brown, T. H., D. H. Perkel, and M. W. Feldman (1976) Evoked neurotransmitter release: Statistical effects of nonuniformity and nonstationarity. Proc. Natl. Acad. Sci. USA 73: 2913-2917.

Clayton, R. W., and T. J. Ulrych (1977) A restoration method for impulse functions. IEEE Trans. Information Theory IT-23: 262-264.

Clements, J. D., I. D. Forsythe, and S. J. Redman (1986) Presynaptic inhibition of synaptic potentials evoked in cat spinal motoneurones by impulses in single group Ia axons. J. Physiol. (Lond.) (in press).

d'Alonzo, A. J., and A. D. Grinnell (1985) Profiles of evoked release along the length of frog motor nerve terminals. J. Physiol. (Lond.) 359: 235-258.

del Castillo, J., and B. Katz (1954) Quantal components of the endplate potential. J. Physiol. (Lond.) 124: 560-573.

Edwards, F. R., S. J. Redman, and B. Walmsley (1976) Statistical fluctuations in charge transfer at Ia synapses on spinal motoneurones. J. Physiol. (Lond.) 259: 665-688.

Fyffe, R. E. W., and A. R. Light (1984) The ultrastructure of group Ia afferent fiber synapses in the lumbosacral spinal cord of the cat. Brain Res. 300: 201-209.

Hines, W. W., and D. C. Montgomery (1972) Probability and Statistics in Engineering and Management Science, Ronald Press, New York.

Houchin, J., D. J. Maxwell, R. E. W. Fyffe, and A. G. Brown (1983) Light and electron microscopy of dorsal spinocerebellar tract neurones in the cat: An intracellular horseradish peroxidase study. Q. J. Exp. Physiol. 68: 719-732.

Jack, J. J. B., S. J. Redman, and K. Wong (1981) The components of synaptic potentials evoked in cat spinal motoneurones by impulses in single group Ia afferents. J. Physiol. (Lond.) 321: 65-96.

Korn, H., A. Triller, A. Mallart, and D. S. Faber (1981) Fluctuating responses at a central synapse: $n$ of binomial fit predicts number of stained presynaptic boutons. Science (N.Y.) 213: 898-901.

Kowalik, J., and M. R. Osborne (1968) Methods for Unconstrained Optimization Problems, Elsevier, New York.

Kuno, M. (1964) Mechanisms of facilitation and depression of the excitatory synaptic potential in spinal motoneurones. J. Physiol. (Lond.) 175: $100-112$.
Kuno, M., and J. T. Miyahara (1969) Analysis of synaptic efficacy in spinal motoneurones from 'quantum' aspects. J. Physiol. (Lond.) 201: 479-493.

Ling, L., and D. J. Tolhurst (1983) Recovering the parameters of finite mixtures of normal distributions from a noisy record: An empirical comparison of different estimating procedures. J. Neurosci. Methods 8: 309-333.

Martin, A. R. (1977) Junctional transmission. II. Presynaptic mechanisms. In The Handbook of Physiology, Sect. I. The Nervous System, Vol. 1, Pt. 1, E. R. Kandel, ed., pp. 329-355, American Physiological Society, Bethesda, MD.

Maxwell, D. J., B. A. Bannatyne, R. E. W. Fyffe, and A. G. Brown (1982) Ultrastructure of hair follicle afferent terminations in the spinal cord of the cat. J. Neurocytol. 11: 571-582.

Maxwell, D. J., B. A. Bannatyne, R. E. W. Fyffe, and A. G. Brown (1984) Fine structure of primary axon terminals projecting from rapidly adapting mechanoreceptors of the toe and foot pads of the cat. Q. J. Exp Phys. 69: 381-392.

McLachlan, E. M. (1978) The statistics of transmitter release at chemical synapses. In International Review of Physiology, Neurophysiology III, 17, R. Porter, ed., pp. 49-117, University Park Press, Baltimore, MD.

Mendell, L. M., and R. Weiner (1976) Analysis of pairs of individual Ia EPSPs in single motoneurones. J. Physiol. (Lond.) 255: 81-104.

Redman, S. J., and B. Walmsley (1981) The synaptic basis of the monosynaptic stretch reflex. Trends Neurosci. 4: 248-250.

Redman, S. J., and B. Walmsley (1983) Amplitude fluctuations in synaptic potentials evoked in cat spinal motoneurones at identified group Ia synapses. J. Physiol. (Lond.) 343: 135-145.

Semba, K., P. Masarachia, S. Malamed, M. Jacquin, S. Harris, G. Yang, and M. D. Egger (1985) An electron microscopic study of terminals of rapidly adapting mechanoreceptive afferent fibers in the cat spinal cord. J. Comp. Neurol. 232: 229-240.

Tracey, D. J., and B. Walmsley (1984) Synaptic input from identified muscle afferents to neurones of the dorsal spinocerebellar tract in the cat. J. Physiol. (Lond.) 350: 599-614.

Walmslcy, B., E. Wieniawa-Narkiewicz, and M. J. Nicol (1985) The ultrastructural basis for synaptic transmission between primary muscle afferents and neurons in Clarke's column of the cat. J. Neurosci. 5: 2095-2106.

Walmsley, B., F. R. Edwards, and D. J. Tracey (1986) An unconstrained quantal model of synaptic transmission between single primary afferent fibres and dorsal spinocerebellar tract neurones in the cat spinal cord. Proc. Aust. Physiol. Pharmacol. Soc. 17(2): 112P.

Walmsley, B., E. Wieniawa-Narkiewicz, and M. J. Nicol (1987) Ultrastructural evidence related to presynaptic inhibition of primary muscle afferents in Clarke's column of the cat. J. Neurosci. 7: 236243.

Wong, K., and S. J. Redman (1980) The recovery of a random variable from a noisy record with application to the study of fluctuations in synaptic potentials. J. Neurosci. Methods 2: 389-409. 